\title{
DIAGNOSIS OF OBSTRUCTION IN EXPERIMENTAL HYDROURETERONEPHROSIS
}

STEPHEN A. KOFF, M.D.

JAMES H. THRALL, M.D.

From the Section of Urology, Department of Surgery, and the Section of Nuclear Medicine, Department of Internal Medicine, University of Michigan Medical Center, Ann Arbor, Michigan

\begin{abstract}
A canine model with partial ureteral obstruction and progressive hydroureteronephrosis was utilized to validate and compare the accuracy of several diagnostic methods currently used to assess urinary tract obstruction. Tests of renal function and measurements of intrapelvic pressure were not helpful in following the progress of hydroureteronephrosis but did provide insight into the mechanisms by which incomplete ureteral occlusion impairs renal function. Serial intravenous pyelography, diuretic radionuclide urography, and perfusion pressure flow studies were frequently accurate in assessing obstruction, but each was subject to potential and, at times, regular misinterpretation. In applying the results of these laboratory studies to the problems of evaluating human hydroureteronephrosis, the clinician must recognize the pitfalls and limitations of these methods in order to ensure an accurate diagnosis of partial urinary obstruction.
\end{abstract}

Complete scientific validation of diagnostic tests which supposedly distinguish between obstructive and nonobstructive hydroureteronephrosis ultimately depends on not operating on kidneys which satisfy the criteria for obstruction and observing their progressive deterioration. Such a planned prospective protocol is obviously unconscionable in humans but is feasible in laboratory animals. Herein, we present an evaluation of several diagnostic methods for assessing obstruction in the dilated urinary tract using a canine model with partial ureteral obstruction.

Material and Methods

Incomplete ureteral ligation was performed in 12 adult female mongrel dogs weighing 15 to 25 $\mathrm{Kg}$. under intravenous sodium pentobarbital anesthesia. The ureter was isolated transperitoneally, approximately 2 to $4 \mathrm{~cm}$. from the ureterovesical junction and partially occluded using a 2-0 silk ligature. An 18-gauge needle was placed alongside the ureter during ligation and thereafter removed to ensure that the ligation was incomplete. A constriction, but not a complete occlusion of the ureter, was produced by this technique. In 6 dogs contralateral nephrectomy also was performed.

The animals were studied pre- and postoperatively at approximately two-week intervals until either the renal pelvic volume exceeded $100 \mathrm{ml}$. or there was clinical evidence of uremia. Deligation of the partially obstructed ureter was then accomplished by surgically removing the silk ligature. Thereafter the animals were again studied at periodic intervals.

The progress of hydronephrosis was assessed in all dogs by the following methods: serum renal function tests (blood urea nitrogen and creatinine); intravenous pyelography including delayed filming; diuretic radionuclide urography; ${ }^{1}$ urodynamic tests (resting intrapelvic pressure, resting intrapelvic volume, and perfusion pressure-flow studies ${ }^{2}$ ). All testing except biochemical study was performed under intravenous sodium pentobarbital anesthesia. A ten-day course of parenteral antibiotics (chloramphenicol $50 \mathrm{mg} . / \mathrm{Kg}$.) was used after each operative study session. 

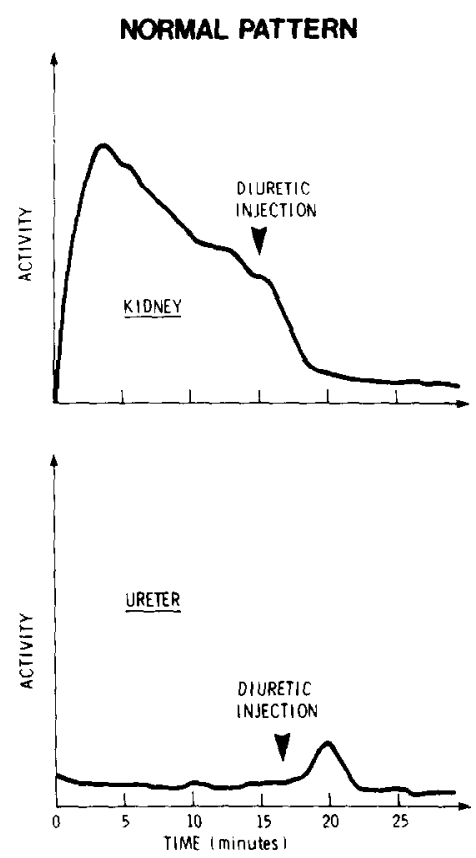

DILATED NON-OBSTRUCTED

PATTERN
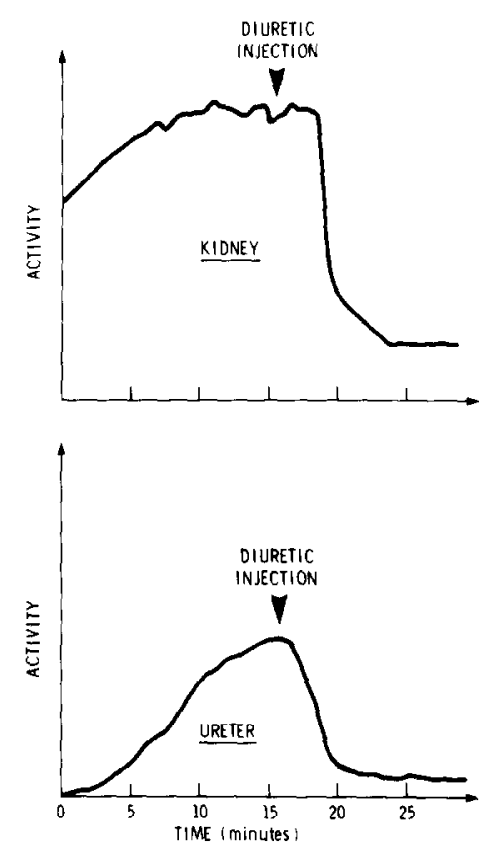

OBSTRUCTED PATTERN
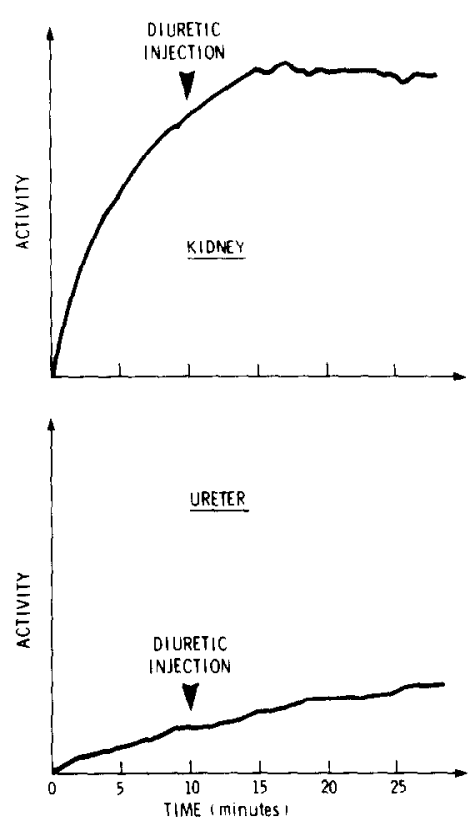

FIGURE 1. Diuretic radionuclide urography. Time activity curves for kidney and ureter illustrating normal, dilated nonobstructed, and obstructed patterns.

The technique of diuretic radionuclide urography has been presented in detail previously.' Briefly, each dog received intravenously a weight-adjusted dose of ${ }^{99} \mathrm{~m}$ technetium-labeled DTPA, $0.14 \mathrm{mCi} / \mathrm{Kg}$., and was studied in the prone position using the gamma scintillation camera. After tracer injection the gamma camera oscilloscope and scintiphotos were monitored to determine when the renal collecting structures (pelvis and calyces) were visualized fully at which point furosemide, $0.3 \mathrm{mg} . / \mathrm{Kg}$., was injected intravenously. Data collection was continued for an additional fifteen minutes. After all data were collected on the nuclear medicine minicomputer (two frames per minute), the entire study was inspected frame by frame to select appropriate frames for region of interest assignment. The kidney regions of interest encompassed the entire kidney including the pelvis but not the ureter which was assigned a separate region. Background corrected time activity curves for each kidney and ureter were then generated and compared with the previously reported normal, dilated but not obstructed, and obstructed patterns (Fig. 1). ${ }^{1}$

The kidney was exposed but not mobilized via an upper midline or flank incision for each set of urodynamic studies. A balloon-type urethral catheter was inserted into the bladder and after emptying the bladder was connected to a water manometer which recorded intravesical pressure.

Resting intrapelvic pressure was measured in a state of antidiuresis using an inertia-free system which employed a 16-gauge Silastic needle catheter (Abbocath) connected to a commercial recording carbon dioxide cystometer with a continuous flow rate of $5 \mathrm{ml}$. per minute. With the gas flowing, the needle catheter was thrust into the renal pelvis through the renal parenchyma and the intrapclvic pressure was recorded. In some cases with high intrapelvic pressure, a small amount of urine would backfill the catheter lumen following intrapelvic placement. In these cases, the value for resting intrapelvic pressure was the pressure recorded after the urine $(<1 \mathrm{cc}$.) had been replaced into the pelvis.

After measurement of intrapelvic pressure, all urine contained within the kidney, renal pelvis, and ureter was aspirated through the needle catheter and the volume was recorded as the pelviureteric volume. Volume was also measured in six nonobstructed nephroureteral systems at the time of nephrectomy.

Perfusion pressure flow studies were performed and repeated according to the methods described by Whitaker ${ }^{2}$ by perfusing saline through the needle catheter at a rate of 10 $\mathrm{ml} . / \mathrm{min}$. using a Harvard pump. The infusion 


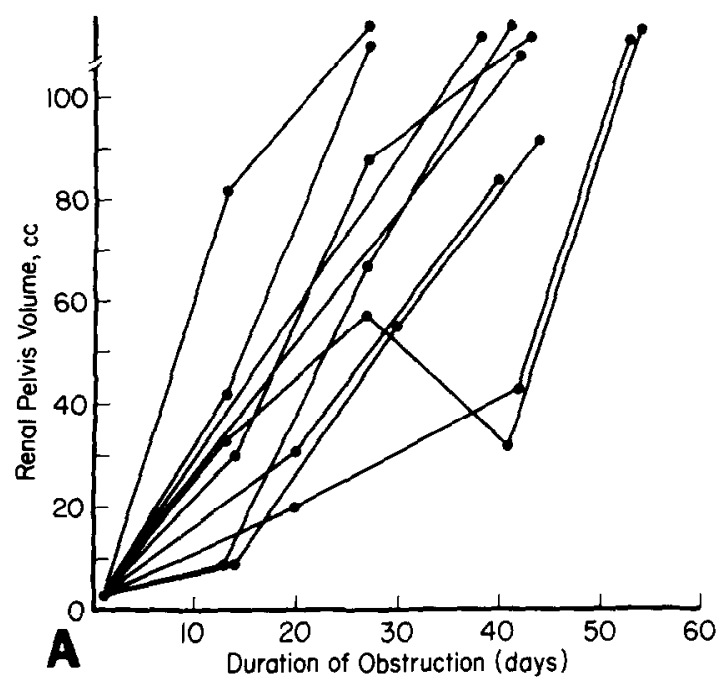

FIGURE 2. (A) Pelviureteric volume after partial ureteral ligation. (B) Effect of partial ureteral ligation and contralateral nephrectomy on renal function. (C) Time course of resting intrapelvic pressure after partial ureteral ligation.

pressure was monitored with an in-line water manometer, and after correcting for intravesical pressure and the measured internal resistance of the system the actual perfusion pressure was obtained and recorded. In all instances, the saline infusion was continued for several minutes after the volume of perfusate instilled equaled the previously measured pelviureteric volume. After completing the perfusion pressure flow study, the pelviureteric volume was again measured.

\section{General}

\section{Results}

Incomplete ureteral ligation by the technique described herein effectively produced a progressive hydroureteronephrosis in all of the experimental animals. In 2 dogs with contralateral nephrectomy, rapidly progressive uremia and death occurred prior to urodynamic testing. Deligation was performed in 10 animals, and 1 died before upper tract urodynamics could be assessed. Hydroureteronephrosis improved in all but 1 dog after deligation where obstruction persisted because of urinary extravasation at the site of ureteral ligature. Pyohydronephrosis occurred in 2 dogs who survived after deligation and antibiotics.

\section{Renal pelviureteric volume}

The volume of urine contained in the pelviureteric systems of the 6 normal nondilated nephrectomized kidneys ranged from 3 to $5 \mathrm{cc}$.
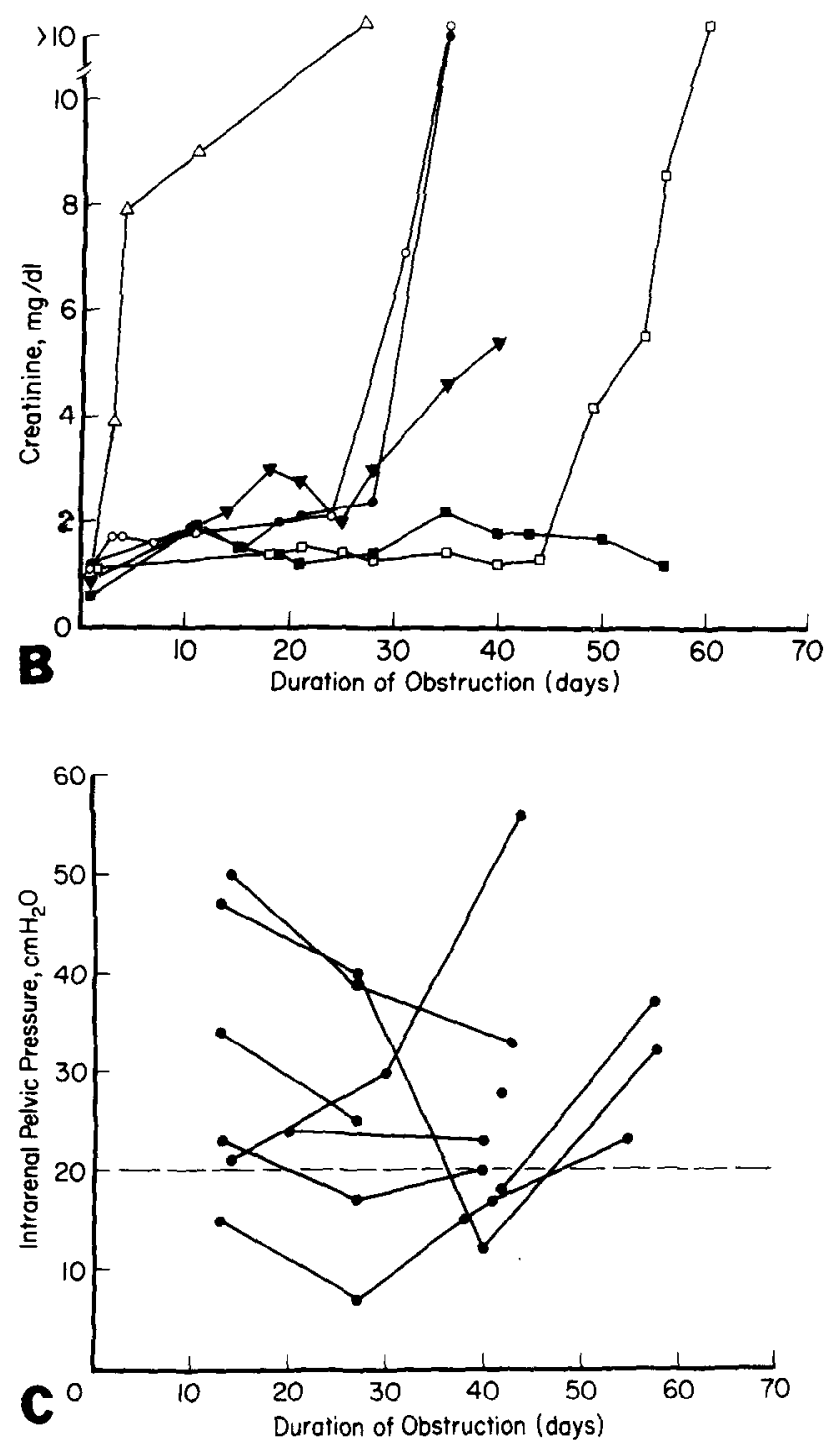

Thirty-seven measurements of renal pelviureteric volume were performed in 10 animals during the development of hydroureteronephrosis and are recorded in Figure 2A. A nearly linear progressive increase in measured volume occurred in all dogs. At the time of deligation the volumes ranged from 81 to $512 \mathrm{cc}$., with an average volume of $241 \mathrm{cc}$.

After successful deligation in 9 survivors, 7 pelviuretcric systems returned to a resting volume of $10 \mathrm{cc}$. or less, while 2 systems remained dilated. In one of the persistently dilated systems $(75 \mathrm{cc}$.) obstruction from urinary extravasation was demonstrated; in the other dilated system $(37 \mathrm{cc}$.) no obstruction was evident.

\section{Renal function studies}

In 6 dogs with partial ureteral ligation and contralateral nephrectomy, 112 separate renal 
Figure 3. Intravenous pyelographic study of canine kidney: (A) preoperatively; (B) two weeks after partial ureteral ligation; $(C)$ one month later there is nonvisualization; and (D) two weeks after deligation - note similar degrees of hydroureteronephrosis before (B) and after (D) deligation.

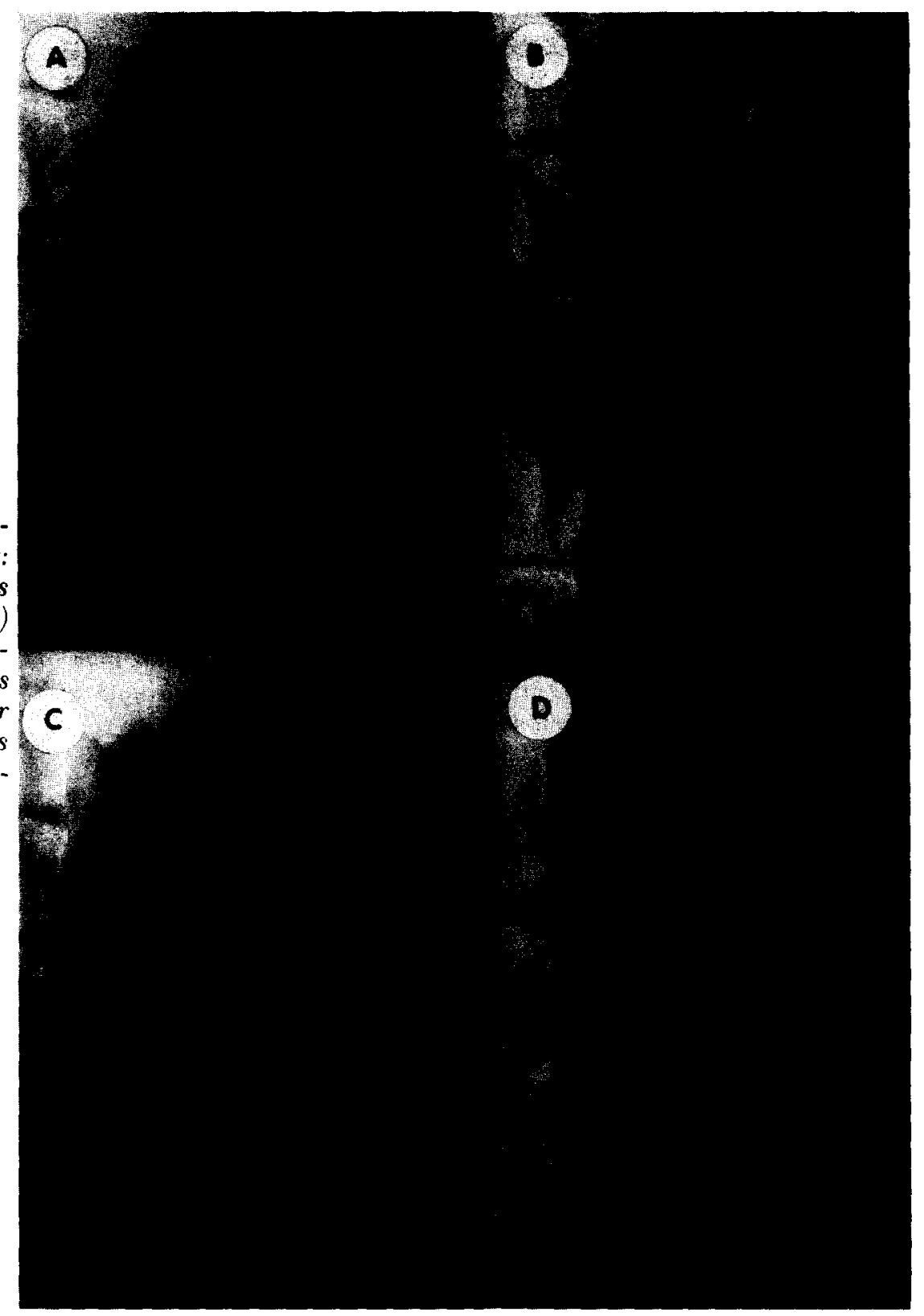

function studies were performed. Control preoperative values for serum creatinine ranged from 0.6 to $1.2 \mathrm{mg} . / \mathrm{dl}$. (mean, 1.0) and blood urea nitrogen ranged from 10 to $21 \mathrm{mg} . / \mathrm{dl}$. (mean, 15).

Figure 2B illustrates the patterns of serum creatinine elevation in dogs with one kidney during the development of hydroureteronephrosis. Except for $1 \mathrm{dog}$ with a rapid impairment in renal function, the remaining animals displayed a plateau of slightly elevated creatinine values for days and weeks. Thereafter, rapidly increasing serum creatinine values occurred abruptly in all but 1 animal. The changes in blood urea nitrogen paralleled those of serum creatinine. An insufficient number of renal function determinations was performed after deligation for analysis.

\section{Intrapelvic pressure}

Thirty-seven resting intrapelvic pressure measurements (IPP) were made during progressive hydroureteronephrosis. The alterations in IPP were highly individualized and variable, and no trend in pressure changes emerged (Fig. 2C). Mean pressure at two, four, and six weeks following obstruction was not significantly different $\left(31,26\right.$, and $27 \mathrm{~cm} . \mathrm{H}_{2} \mathrm{O}$, respectively). 


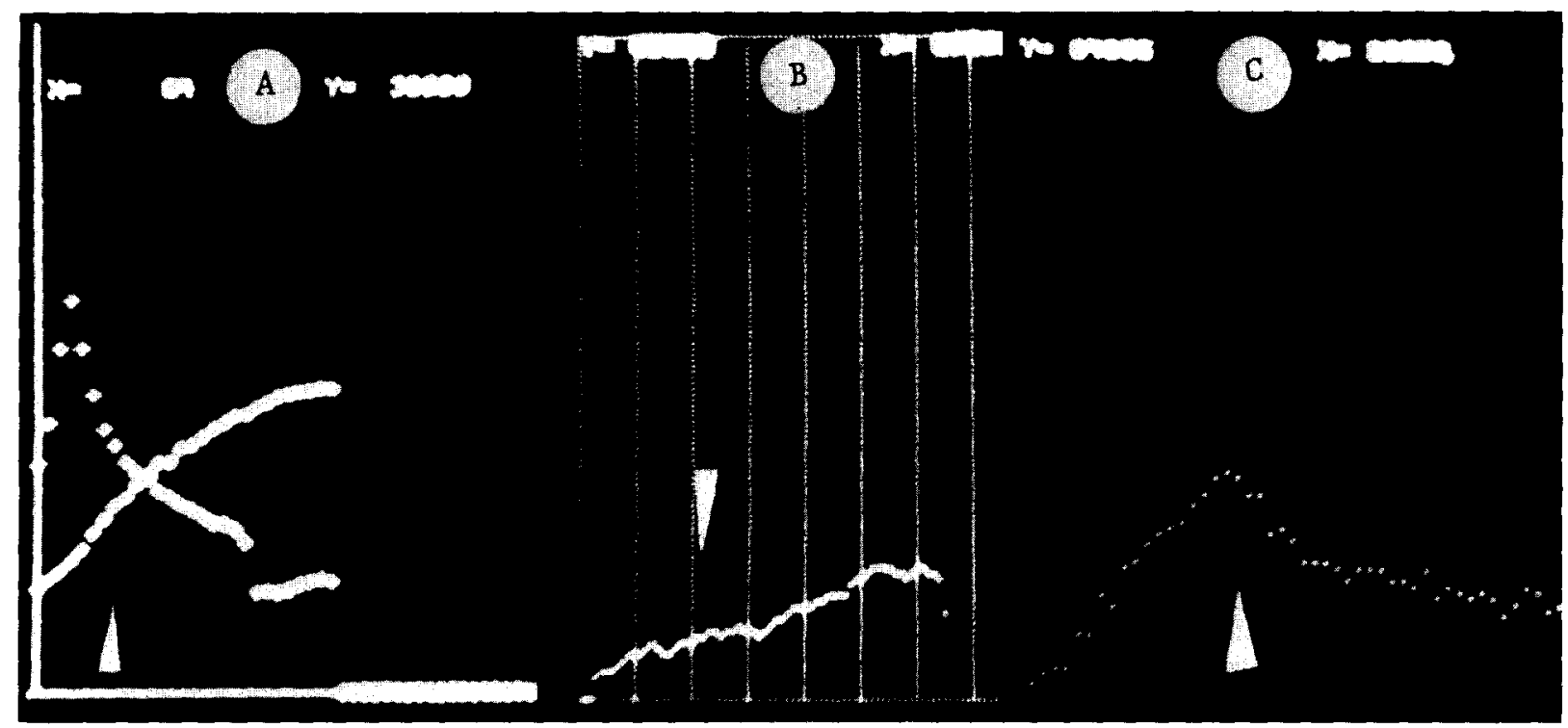

FIGURE 4. Diuretic radionuclide urographic study of canine kidney after partial ureteral obstruction. Arrows indicate time of diuretic injection: (A) two weeks after ligation obstructed kidney demonstrates obstructed pattern, contralateral kidney pattern is normal; $(B)$ one month later obstructed pattern persists; and $(C)$ two weeks after deligation dilated nonobstructed pattern is registered.

The upper limit of normal for resting IPP was $20 \mathrm{~cm} . \mathrm{H}_{2} \mathrm{O}(15.4 \mathrm{~mm}$. $\mathrm{Hg})$. In hydronephrotic systems with ureteral obstruction, the resting IPP was observed to be within the normal range in 8 of 25 measurements in 5 animals. After release of obstruction IPP was normal $(15 \mathrm{~cm}$. or less) in all animals including the dog with documented persistent hydronephrosis.

\section{Intravenous pyelography}

Intravenous pyelography (IVP) including delayed filming (two hours) was performed preoperatively and at intervals postoperatively in all dogs (46 studies). Preoperative examinations were normal in each animal. Progressive hydroureteronephrosis developed in all dogs, and at the time of deligation, nonvisualization of the affected kidney occurred in 8 dogs. Improvement in the IVP was observed transiently in onc obstructed kidney, and this was associated with a transient reduction in resting pelviureteric volume. Deligation of the ureter was followed by pyelographic improvement in 8 dogs while one remained obstructed (Fig. 3).

\section{Diuretic radionuclide urography}

One hundred nine diuretic radionuclide urographic studies were performed including 31 examinations of normal nonobstructed canine kidneys and ureters (Fig. 4). In all $10 \mathrm{dogs}$ with obstructed ureters, the diuretic radionuclide ureteral study demonstrated an obstructed pat- tern in each of 24 examinations. The corresponding diuretic radionuclide renal study, however, showed an obstructed pattern in only 20 of the 24 studies, and in 4 instances the renal histogram registered a dilated but nonobstructed pattern. All 4 of these kidneys were visibly hydronephrotic and serum creatinine values ranged between 1.4 and $2.8 \mathrm{mg} . / \mathrm{dl}$. (mean, $2.1 \mathrm{mg} . / \mathrm{dl}$.).

After deligation of the ureter in 9 surviving animals, renal and ureteral diuretic radionuclide studies ultimately showed a dilated but nonobstructed pattern in 6 of the dogs. The average length of time required after deligation for the diuretic radionuclide study to begin displaying washout was five weeks, with a range of two to twelve weeks. Renal and ureteral diuretic radionuclide studies in 2 dogs without obstruction continued to demonstrate an obstructed pattern; a similar pattern also occurred in a third animal with persisting obstruction due to urinary extravasation.

\section{Perfusion pressure flow studies}

Forty perfusion pressure flow studies were performed, and a perfusion pressure of greater than $22 \mathrm{~cm}$. $\mathrm{H}_{2} \mathrm{O}$ was used to define an obstructed system (Fig. 5). Pressures less than $15 \mathrm{~cm} . \mathrm{H}_{2} \mathrm{O}$ were considered normal and values in between, equivocal. During progressive hydroureteronephrosis, pressures in the obstructed range occurred in 20 studies in 6 
dogs, while pressures in the equivocal range ( 15 to $22 \mathrm{~cm} . \mathrm{H}_{2} \mathrm{O}$ ) were noted in 6 studies in 4 dogs. After deligation with release of obstruction the perfusion pressures were in the normal range in 12 studies in 7 dogs, and in the obstructed range in 2 studies in 2 dogs, one of which occurred in the persistently hydronephrotic system.

After completing perfusion pressure flow studies in 3 dogs whose pressures equilibrated in the nonobstructed range, the entire pelviureteric system was aspirated and the volume measured. The recovered volumes represented 84 to 95 per cent of the volumes of perfusate instilled during the perfusions.

\section{Comment}

The aims of this study were first to produce a canine model with partial ureteral obstruction and progressive hydroureteronephrosis, and then to examine the accuracy and identify the pitfalls of several clinical diagnostic methods currently used to assess obstruction in dilated kidneys and ureters. We were successful in producing an incomplete obstruction by using a partially occluding ligature, but the degree of obstruction was variable; upper tract dilatation proceeded at different rates, and 3 dogs died before all testing was complete. Progression of hydroureteronephrosis occurred in all kidneys with partially ligated ureters and was documented by serial measurements of enlarging pelviureteric volumes. That the obstruction was incomplete was confirmed by recovering perfusate from the urinary bladder following pressure flow studies. Additionally, the rate at which the pelviureteric systems expanded served as a time frame on which to evaluate the other diagnostic tests.

Although serum renal function studies are often used clinically to follow patients with hydroureteronephrosis, they were not reliable in assessing the progress of canine urinary tract obstruction. Sequential measurements in onekidney dogs with partial ureteral occlusion clearly indicated that mild elevations of serum creatinine ( 1.4 to $2.2 \mathrm{mg} . / \mathrm{dl}$.) and blood urea nitrogen could be maintained while the upper tract progressively dilated. Consequently, elevations of serum creatinine in this range were of no value in following hydronephrosis to determine if progressive changes were occurring nor in predicting whether or when severe renal insufficiency would ensue. Likewise, persistence of normal or minimally elevated serum creatinine levels for a period of weeks was no insurance that renal function would not abruptly worsen.

Canine intravenous pyelograms were faithful in registering progression of hydroureteronephrosis, but similar degrees of dilation were regularly observed in the same renal unit before and after deligation. Therefore, each individual intravenous pyelographic study was of no value in predicting whether hydroureteronephrosis was getting better or worse. However, when the intravenous pyelograms in each animal were viewed as a collective series, they were consistently effective in all dogs in chronologizing pelviureteric volume changes, and were accurate in determining whether hydroureteronephrosis was progressing or improving. An exception to this occurred when poor renal function and pyelographic nonvisualization made comparisons between kidneys impossible.

Although elevated intrapelvic pressure is considered to be pathogenetic in producing hydronephrotic renal damage, measurements of pressure in chronic hydronephrosis are often normal. ${ }^{3-6}$ The reported time course of intrapelvic pressure changes following total canine ureteral occlusion shows a return from elevated to normal pressures within days or weeks; ${ }^{7}$ this reflects a severe impairment in renal function with a resulting inability to generate high hydrostatic pressures. ${ }^{8}$ Previous reports of intrapelvic pressure measurements in animals with partial ureteral obstruction have described a similar trend toward normal pressures with duration of obstruction. ${ }^{6,9-11}$ This appears paradoxical because renal function is usually less severely affected during experimental partial obstruction, and elevated intrapelvic pressures should be registered at least intermittently. An alternative explanation in such cases is that sustained normal intrapclvic pressure during partial occlusion reflects an insufficient degree of obstruction to produce a progressive impairment in renal function. This is supported by noting that although an initial deterioration in renal function and/or hydronephrosis occurred in many of the reported animals with partial obstruction, true progression of these abnormalities rarely was documented. ${ }^{6,9,11,12}$

In contrast, our study animals with documented progressive hydroureteronephrosis demonstrated no tendency for normalization of intrapelvic pressures which fluctuated widely as a function of time. We contend that this seemingly erratic intrapelvic pressure response 


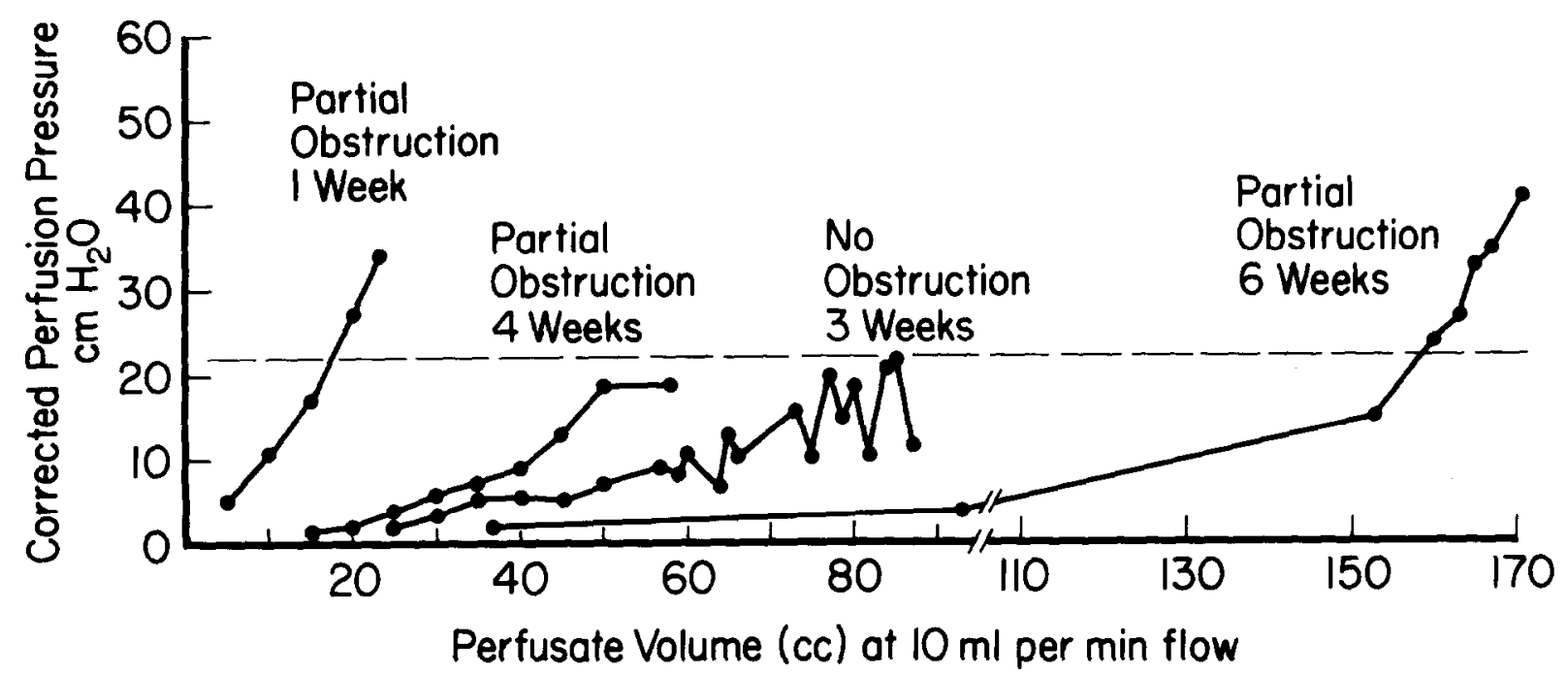

Figure 5. Potential for error is illustrated in perfusion pressure flow studies performed sequentially in a canine kidney subjected to partial ureteral ligation and then deligation. Pelviureteric filling and volume expansion occurs at nommal pressures. If large dilated system is not filled to capacily, equilibration of pressures in normal range may simulate no obstruction. After release of obstruction, vigorous pelviureteric peristalsis may artificially elevate recorded pressures.

to partial ureteral obstruction actually reflects periodic overdistention of the renal pelvis which produces and directly identifies elevated pressures in the pathogenesis of hydronephrotic renal damage. This repetitive process of overdistention of the renal pelvis with measurably increased pressures and impaired renal function, followed by gradual emptying, and then refilling and improvement in renal function is probably the common mechanism by which all incompletely obstructed nephroureteral systems undergo progressive and sizeable hydronephrotic dilation while still preserving renal function. ${ }^{3}$ Eventually if an equilibrium state is not reached, the additive effects of intermittent raised intrapelvic pressures would cause renal function to deteriorate, at times abruptly, as it did in our experimental animals.

Intrapelvic pressure was normal at some time during the progression of hydroureteronephrosis in half of the experimental animals. Measurement of pressure, therefore, was of limited value in predicting whether or not urinary tract dilation was progressive.

Canine perfusion pressure flow studies, performed according to Whitaker's technique and criteria, ${ }^{2}$ were able to identify obstruction in only 6 of 10 dogs in 20 of 26 studies. Overlap in perfusion pressures between normal and partially obstructed ureters has been previously reported in experimental animals. ${ }^{13}$ After relief of obstruction, however, the test was more successful in recognizing a nonobstructed system.
In attempting to identify a cause for these falsely negative results, we observed that large volumes of perfusate were recovered by aspiration from the obstructed pelviureteric systems of those dogs whose perfusion pressures equilibrated in the nonobstructed range ( 84 to 95 per cent of the instilled perfusate). This finding indicates that while there was filling of the renal pelvis and ureter, little fluid passed across the site of obstruction, and this is at variance with the original premise that "the pressure which is recorded is that pressure neccssary to drive the fluid through the system at a fixed flow rate."2 Examination of pressure flow studies in each animal at periodic intervals supported the fact that pelviureteric filling and volume expansion may occur at normal pressures without propulsion of significant amounts of fluid across the ureteral obstruction; the pressures recorded were filling pressures rather than true pressure flow values. This may simulate equilibration of pressures in the normal or nonobstructed range and result in a falsely negative test interpretation (Fig. 5). To remedy this potential problem, especially in large volume dilated systems, perfusion must be continued for a sufficiently long enough period of time to fill the pelvis and ureter to their physiologic capacity before normal pressures are accepted as validly indicating absence of obstruction.

Previous reports on the use of diuretic radionuclide urography have emphasized that the ureteral histogram is more accurate than is 
the renal histogram in assessing ureteral obstruction. ${ }^{1,14}$ This was confirmed in our study: during obstruction all ureteral histograms demonstrated obstruction, whereas 4 of 24 renal histograms showed no obstruction. For maximum accuracy, therefore, regions of interest must be selected from the area of dilation nearest and proximal to the site of obstruction, in this case the distal ureter.

Soon after release of obstruction the diuretic radionuclide urogram was temporarily inaccurate and often did not demonstrate washout of tracer until sufficient time had elapsed (two to twelve weeks) for renal function to recover to a level where furosemide injection would produce a diuresis. In 2 animals, however, the renal and ureteral histograms continued to demonstrate an obstructed pattern in the absence of organic obstruction, but in both instances a severe renal functional impairment persisted.

In attempting to extrapolate from these canine studies to human hydronephrosis, one must consider carefully that renal pathologic changes develop and resolve much more rapidly in the dog than in man. As a result, alterations that occur abruptly in the dog may progress at a slower rate in the human kidney, while those changing slowly in the canine may be altered imperceptibly or not at all in man. Any of the diagnostic studies, therefore, which depend on serial changes such as serum renal function study and intrapelvic pressure and volume may be less accurate in man because the changes occur more slowly; therefore, the results of those studies must be extrapolated with caution from canine to man.

The three most accurate methods for assessing canine ureteral obstruction were serial intravenous pyelography, diuretic radionuclide urography, and perfusion pressure flow studies. In human investigations, the intravenous pyelogram is consistently less helpful because often there is only a single examination, and the risk of waiting for evidence of progressive destruction is usually not acceptable, especially in children. The latter two methods, however, have been shown to be particularly effective in investigating hydronephrosis. In examining their tendencies for falsely positive or negative results, we find that the diuretic radionuclide urogram and the perfusion pressure flow examinations are complementary in clinical practice: when impaired renal function prevents tracer washout for lack of a diuresis, the perfusion study is unaffected; and when perfusion pressures appear to be normal in large capacity obstructed systems, choice of a region of interest near the obstruction will often make the radionuclide study more accurate.

In practice, these diagnostic methods, when used individually or in combination, are in a sense only laboratory tests which provide different bits of data regarding the anatomy, function, and kinetics of the dilated urinary tract suspected of being obstructed. By recognizing the pitfalls and limitations of each study and integrating the results rationally, the clinician can usually, but not always, arrive at a correct diagnosis. Until we better understand and can measure specifically the factors actually responsible for progressive renal deterioration in cases of incomplete obstruction, we will have to settle for these reliable empiric studies and accept an occasional measure of inaccuracy.

University Hospital, Box 03

Ann Arbor, Michigan 48109

(DR. KOFF)

References

1. Koff SA, Thrall JH, and Keyes JW, Jr: Diuretic radionuclide urography: a non-invasive method for evaluating nephroureteral dilation, J. Urol. 122: 451 (1979).

2. Whitaker RH: Methods of assessing obstruction in dilated ureters, Br. J. Urol. 45: 15 (1973).

3. Hinman F, Jr: The pathophysiology of urinary obstruction, in Campbell MF, and Harrison JH (Eds): Urology, Philadelphia W.B. Saunders Company, 1970.

4. Backlund L, Grotte G, and Reuterskiold A: Functional stenosis as a cause of pelvi-ureteric obstruction and hy. dronephrosis, Arch. Dis. Child. 40: 203 (1965).

5. Djurhuus $\mathrm{JC}$, and Stage $\mathrm{P}$ : Percutaneous intrapelvic pressure registration in hydronephrosis during diuresis, Acta Chir. Scand. 472: 43 (1976).

6. Schweitzer FAW: Intrapelvic pressure and renal function studies in experimental chronic partial ureteric obstruction, Br. J. Urol. 45: 2 (1973).

7. Vaughn ED, Jr, Sorenson EJ, and Gillenwater JY: The renal hemodynamic response to chronic unilateral complete ureteral occlusion. Invest. Urol. 8: 78 (1970).

8. Rose JG, and Gillenwater JY: Effects of obstruction on ureteral function, Urology 12: 139 (1978).

9. Djurhuus JC, Nerstrom B, Gyrd-Hansen N, and RaskAndersen H: Experimental hydronephrosis, Acta Chir. Scand 472: 17 (1976).

10. Weaver RG: Reabsorptive patterns and pressures in hydronephrosis with clinical application, J. Urol. 100: 112 (1968).

11. Boyarsky S, and Martinez J: Pathophysiology of the ureter: partial ligation of the ureter in dogs, Invest. Urol. 2: 173 (1964).

12. Olesen S, and Madsen PO: Function during partial obstruction following contralateral nephrectomy in the dog, J. Urol. 99: $692(1968)$.

13. Koff SA: Experimental assessment of graded ureteral obstruction utilizing liquid and gaseous perfusion techniques, Invest. Urol. 16: 229 (1978).

14. Koff SA, Thrall JH, and Keyes JW, Jr: Assessment of hydroureteronephrosis in children utilizing diuretic radionuclide urography, J. Urol. 123: 531 (1980). 